\title{
Treatment options for polycystic ovary syndrome
}

This article was published in the following Dove Press journal:

International Journal of Women's Health

8 February 20II

Number of times this article has been viewed

\author{
Ahmed Badawy' \\ Abubaker Elnashar ${ }^{2}$ \\ 'Department of Obstetrics and \\ Gynecology, Mansoura University, \\ Mansoura, Egypt; ${ }^{2}$ Department of \\ Obstetrics and Gynecology, Benha \\ University, Benha, Egypt
}

Correspondence: Ahmed Badawy Department of Obstetrics and Gynecology, Mansoura University, PO 35 I I I, Mansoura, Egypt $\mathrm{Tel}+20502100101$

Fax +20 502303939

Emailambadawy@yahoo.com

\begin{abstract}
Polycystic ovary syndrome (PCOS) is the most common endocrine disorder in women. The clinical manifestation of PCOS varies from a mild menstrual disorder to severe disturbance of reproductive and metabolic functions. Management of women with PCOS depends on the symptoms. These could be ovulatory dysfunction-related infertility, menstrual disorders, or androgen-related symptoms. Weight loss improves the endocrine profile and increases the likelihood of ovulation and pregnancy. Normalization of menstrual cycles and ovulation could occur with modest weight loss as little as $5 \%$ of the initial weight. The treatment of obesity includes modifications in lifestyle (diet and exercise) and medical and surgical treatment. In PCOS, anovulation relates to low follicle-stimulating hormone concentrations and the arrest of antral follicle growth in the final stages of maturation. This can be treated with medications such as clomiphene citrate, tamoxifen, aromatase inhibitors, metformin, glucocorticoids, or gonadotropins or surgically by laparoscopic ovarian drilling. In vitro fertilization will remain the last option to achieve pregnancy when others fail. Chronic anovulation over a long period of time is also associated with an increased risk of endometrial hyperplasia and carcinoma, which should be seriously investigated and treated. There are androgenic symptoms that will vary from patient to patient, such as hirsutism, acne, and/or alopecia. These are troublesome presentations to the patients and require adequate treatment. Alternative medicine has been emerging as one of the commonly practiced medicines for different health problems, including PCOS. This review underlines the contribution to the treatment of different symptoms.
\end{abstract}

Keywords: treatment, polycystic ovary syndrome

\section{Introduction}

Polycystic ovary syndrome (PCOS) is the most common endocrine disorder in women. Its prevalence among infertile women is $15 \%-20 \%$. The etiology of PCOS remains unclear; however, several studies have suggested that PCOS is an X-linked dominant condition. Women with PCOS have abnormalities in the metabolism of androgens and estrogen and in the control of androgen production. High serum concentrations of androgenic hormones, such as testosterone, androstenedione, and dehydroepiandrosterone sulfate (DHEAS), may be encountered in these patients. However, individual variation is considerable, and a particular patient might have normal androgen levels. PCOS is also associated with peripheral insulin resistance and hyperinsulinemia, and obesity amplifies the degree of both abnormalities. Insulin resistance in PCOS can be secondary to a postbinding defect in insulin receptor signaling pathways, and elevated insulin levels may have gonadotropin-augmenting effects on ovarian function. In addition, insulin resistance in PCOS has been associated 
with adiponectin, a hormone secreted by adipocytes that regulates lipid metabolism and glucose levels. Both lean and obese women with PCOS have lower adiponectin levels than women without PCOS. A proposed mechanism for anovulation and elevated androgen levels suggests that under the increased stimulatory effect of luteinizing hormone (LH) secreted by the anterior pituitary, stimulation of the ovarian theca cells is increased. In turn, these cells increase the production of androgens (eg, testosterone, androstenedione). Because of a decreased level of follicle-stimulating hormone (FSH) relative to $\mathrm{LH}$, the ovarian granulosa cells cannot aromatize the androgens to estrogens, which leads to decreased estrogen levels and consequent anovulation. Growth hormone and insulin-like growth factor 1 may also augment the effect on ovarian function. ${ }^{1,2}$

In this review, the state of the art in the treatment of different aspects of PCOS, from anovulation to hyperandrogenism, is discussed, with a particular emphasis on the emerging new modalities of treatment such as alternative therapy.

\section{Diagnosis of PCOS}

The clinical manifestation of PCOS varies from a mild menstrual disorder to severe disturbance of reproductive and metabolic functions. Women with PCOS are predisposed to type 2 diabetes or develop cardiovascular disease. ${ }^{3}$ Factors implicated in the low fertility in these patients include anovulation, increased risk of early miscarriage, and late obstetric complications. Clinical manifestations include menstrual disorders and signs of hyperandrogenism. Although not universal and not part of the definition, insulin resistance and obesity are also extremely common accompaniments of this syndrome. ${ }^{4}$ This phenotypic nonuniformity and the variability of presentation have made it difficult to define the syndrome.

The 1990 National Institutes of Health (NIH)-sponsored conference for definition required oligo-ovulation, clinical or biochemical hyperandrogenism, and the exclusion of other known disorders, such as late-onset congenital adrenal hyperplasia and Cushing's syndrome ${ }^{5}$ (Table 1). The diagnostic criteria of the syndrome were revised by the Rotterdam European Society for Human Reproduction/American Society of Reproductive Medicine (ASRM)-sponsored PCOS consensus workshop group in 2003, where the following criteria were established: oligo/amenorrhea, clinical and biochemical signs of hyperandrogenism, and sonographically confirmed PCOS. ${ }^{6}$ Two of the three criteria are required for diagnosis (after exclusion of other etiologies such as congenital adrenal hyperplasia, androgen-secreting tumors, or Cushing's syndrome). Sonographic features of PCOS
Table I Diagnostic criteria

\begin{tabular}{|c|c|c|}
\hline Organization/group & Year & Criteria \\
\hline $\begin{array}{l}\text { National Institutes } \\
\text { of Health }\end{array}$ & 1990 & $\begin{array}{l}\text { Both hyperandrogenism and } \\
\text { chronic anovulation }^{5}\end{array}$ \\
\hline Rotterdam European & 2003 & Two of the following conditions: \\
\hline Society for Human & & hyperandrogenism, chronic \\
\hline Reproduction/American & & anovulation, polycystic ovary ${ }^{6}$ \\
\hline \multicolumn{3}{|l|}{ Society of Reproductive } \\
\hline \multicolumn{3}{|l|}{ Medicine-sponsored } \\
\hline \multicolumn{3}{|l|}{ PCOS consensus } \\
\hline \multicolumn{3}{|l|}{ workshop group } \\
\hline Androgen Excess & 2006 & Hyperandrogenism and ovarian \\
\hline \multirow[t]{3}{*}{ Society } & & dysfunction (including infrequent \\
\hline & & or irregular ovulation or anovulation) \\
\hline & & and/or polycystic ovary ${ }^{7}$ \\
\hline
\end{tabular}

Abbreviation: PCOS, polycystic ovary syndrome.

include the presence of 12 or more follicles in each ovary measuring 2-9 $\mathrm{mm}$ in diameter and/or increased ovarian volume $(>10 \mathrm{~mL})$. This is regardless of follicle distribution or ovarian stromal echogenicity. One ovary fulfilling this definition is sufficient to define PCOS. ${ }^{2,7}$ It is recognized that some women with sonographic findings of PCOS may have regular cycles without clinical or biochemical signs of hyperandrogenism. Although this has been a practical working definition, others believe that hyperandrogenism should be an integral part of the definition.

Ovulatory women with PCOS seem to be less insulin resistant than anovulatory women with $\mathrm{PCOS} ;{ }^{8}$ further, a study published in 2007 suggests that women with PCOS, ${ }^{9}$ chronic anovulation, and normal androgen levels are not insulin resistant. These observations limit the usefulness of the Rotterdam criteria, and accordingly an expert panel of the Androgen Excess Society (AES) recommended that PCOS should be considered a disorder of androgen excess and that the NIH diagnostic criteria should be used. ${ }^{7}$ The AES also recommended that women with hyperandrogenism, PCOS, and ovulatory cycles should be considered to have a PCOS phenotype; thus, hyperandrogenism and infrequent or irregular ovulation, as well as hyperandrogenism, regular ovulation, and PCOS, fulfill AES criteria for PCOS.

\section{Management of PCOS}

Management of women with PCOS depends on the symptoms. These could be ovulatory dysfunction-related infertility, menstrual disorders, or androgen-related symptoms.

\section{Weight reduction}

There is some evidence that PCOS-related hyperandrogenism causes central obesity with a high waist/hip ratio independent 
of the body mass index (BMI). It is well established that obesity is associated with anovulation, miscarriage, or late pregnancy complications (such as pre-eclampsia and gestational diabetes). ${ }^{10,11}$ Obesity is observed in 35\%-60\% of women with PCOS and is related to lack of or delayed response to different treatments such as clomiphene citrate (CC), gonadotropins, and surgical treatment of diathermy via laparoscopy. ${ }^{12}$

Weight loss improves the endocrine profile and increases the likelihood of ovulation and pregnancy. Normalization of the menstrual cycles and ovulation could occur with modest weight loss as little as $5 \%$ of the initial weight. ${ }^{13}$ Weight loss can improve not only circulating androgen and glucose levels but also ovulation and pregnancy rates in obese women with PCOS; however, weight loss is only recommended for those who are overweight with a BMI $>25-27 \mathrm{~kg} / \mathrm{m}^{2}$. The treatment of obesity includes modifications in lifestyle (diet and exercise) and medical and surgical treatment. All these treatments must be performed during the preconception period and not jointly with reproduction therapies.

\section{Diet}

Diets recommended for obese PCOS patients are low in calories with a reduced carbohydrate intake, and any form of these diets can produce the $5 \%-10 \%$ loss necessary to re-establish ovarian function in these patients. In 2005, Reaven suggested that low-fat diets produce a decrease in hyperinsulinemia, which improves metabolic effects. ${ }^{14}$

\section{Exercise}

Several studies have attempted to establish the role of exercise in the treatment of obese PCOS patients. ${ }^{15}$ None found significant differences when different diets, associated or not with exercise, were compared, although a longer weight loss maintenance time did appear to be associated in these patients. An increase in physical activity is recommended for PCOS patients, although this often presents limitations. ${ }^{16}$ A knowledge gap exists regarding the optimal type, duration, and frequency of exercise.

\section{Bariatric surgery}

Recently, bariatric surgery has been advocated as a strategy for weight loss in the morbidly obese. In addition, if spontaneous weight loss cannot be achieved with diet and exercise, bariatric surgery can be offered. Two primary approaches, restrictive and combined restrictive, and malabsorptive procedures, adjustable gastric banding, and the Roux-en-Y gastric bypass, are commonly performed. Not surprisingly, in 17 women with PCOS and a mean BMI of $50.7 \mathrm{~kg} / \mathrm{m}^{2}$, bariatric surgery resulted in an average loss of $41 \pm 9 \mathrm{~kg}$ in 12 months and improvements in ovulation, insulin resistance, hyperandrogenism, and hirsutism. ${ }^{17}$ In a group of 12 PCOS patients available for follow-up after bariatric surgery for morbid obesity, regular cycles were restored in all. ${ }^{17}$ Of note, women who have had bariatric surgery are at increased risk for nutritional deficiencies, including protein, iron, vitamin $\mathrm{B}_{12}$, folate, vitamin $\mathrm{D}$, and calcium; however, no consensus exists regarding optimal nutritional screening and supplementation.

\section{Ovulation induction}

In PCOS, anovulation relates to low FSH concentrations and the arrest of antral follicle growth in the final stages of maturation. Excess LH, androgens, and insulin may individually or collectively play a direct or indirect role in this process, augmenting steroidogenesis but arresting follicular growth. For many women, anovulatory infertility is the presenting complaint. Medications and other options available for the induction of ovulation are reviewed in the following sections.

\section{$\mathrm{CC}$}

$\mathrm{CC}$ constitutes one of the first-line treatments for ovulation induction in these patients, as it is economical, is straightforward, has few adverse effects, and requires little monitoring. ${ }^{18}$ $\mathrm{CC}$ is an estrogen receptor antagonist that interferes with negative feedback of the estrogen-signaling pathway, resulting in increased availability of FSH. Increased FSH leads to follicular growth, followed by an LH surge and ovulation. CC is indicated in patients with PCOS and anovulation with normal FSH levels, but it has certain limitations in patients with a BMI $>30$ and advanced age. Legro et al found significant differences in pregnancy rates in patients with a BMI $>30$ compared with those with a BMI $<30 .{ }^{19}$

Doses of 50-150 $\mathrm{mg}$ are administered for 5 days, starting on days 3 or 5 of a progestin-induced or spontaneous cycle. CC produces ovulation in $75 \%-80 \%$ of PCOS patients, although when the gestation rate is assessed, it nears $22 \%$ per ovulation cycle. ${ }^{20}$ These differences in results are attributed to the antiestrogenic effects of $\mathrm{CC}$, mainly on the endometrium and the cervical mucus. ${ }^{18}$ The live birth rate following 6 months of clomiphene ranged from $20 \%$ to $40 \%$. Furthermore, the majority of pregnancies occurred within the first six ovulatory cycles following the initiation of treatment. ${ }^{19}$ Multiple pregnancy rates are under $10 \%$, and hyperstimulation syndrome is rare. Tamoxifen is another 
oral ovulatory agent that is similar to $\mathrm{CC}$ in its mechanism of action, but it lacks its antiestrogenic effect on the cervix and endometrium. It can be used as an alternative to $\mathrm{CC}$ in case of CC resistance or failure.

\section{Metformin}

Metformin is a biguanide currently used as an oral antihyperglycemic agent and is approved by the US Food and Drug Administration (FDA) to manage type 2 diabetes mellitus. The use of metformin is associated with increased menstrual cyclicity, improved ovulation, and a reduction in circulating androgen levels. ${ }^{21}$ Metabolic benefits are enhanced in the presence of weight loss, and weight loss itself may be enhanced in the presence of metformin. Its primary clinical action is to inhibit hepatic glucose production, although it also decreases intestinal glucose uptake and increases insulin sensitivity in peripheral tissues. ${ }^{22}$ Metformin likely plays its role in improving ovulation induction in women with PCOS through a variety of actions, including reducing insulin levels and altering the effect of insulin on ovarian androgen biosynthesis, theca cell proliferation, and endometrial growth. In addition, potentially through a direct effect, it inhibits ovarian gluconeogenesis and thus reduces ovarian androgen production.

Several dose regimens have been proposed. ${ }^{23}$ In order to increase patient tolerance, metformin is started at $500 \mathrm{mg}$ daily with food. After 1 week, the dose is increased to $1000 \mathrm{mg}$ for another week and then to $1500 \mathrm{mg}$ daily. The target dose is $1500-2550 \mathrm{mg} /$ day (500 or $850 \mathrm{mg}$ three times daily). Clinical response is usually seen at the dose of $1000 \mathrm{mg}$ daily. It appears that some PCOS patients who do not respond to metformin at a dose of $1500 \mathrm{mg}$ daily will respond favorably to $2000 \mathrm{mg}$ daily. The most common side effects of metformin are nausea and diarrhea. Lactic acidosis has been described mainly in patients with renal impairment, congestive heart failure, and sepsis. Traditionally, oral hypoglycemic agents have been regarded as teratogenic, and their use is contraindicated in pregnancy. However, an increasing amount of data supports their safety when used throughout the pregnancy. Glueck et al reported no major birth defects and no effect on motor or social development of infants at 3 and 6 months of age. ${ }^{24}$ Compared with the control group of women who did not receive metformin, the incidence of gestational diabetes in the treated group was significantly lower.

To define the exact role of metformin in ovulation induction, it is crucial to distinguish two different indications. In naive PCOS, metformin, as compared with placebo, has been shown to improve ovulation rates, but metformin did not exert significant advantage over $\mathrm{CC}$ with respect to cumulative ovulation, pregnancy, or live birth rates. ${ }^{25}$ The combined approach of metformin plus $\mathrm{CC}$ is not better than $\mathrm{CC}$ or metformin monotherapy in naive $\mathrm{PCOS}^{26}$ In CC-resistant patients, metformin has no benefit over placebo in ovulation, pregnancy, and live birth rates as a single agent, but the combination of metformin and CC significantly improved ovulation and pregnancy rates when compared with $\mathrm{CC}$ alone. However, combined therapy did not improve the odds of live birth. ${ }^{27}$ Metformin pretreatment improves the efficacy of CC in PCOS patients with CC resistance. ${ }^{28}$

Troglitazone is another insulin-sensitizing drug that has been shown to improve ovulation and increase pregnancy rates. However, due to its hepatotoxic effect, it has been withdrawn from the market. ${ }^{29}$ Another drug in the same category, rosiglitazone ( $8 \mathrm{mg} /$ day), has been shown to enhance both spontaneous and clomiphene-induced ovulation in women with PCOS with a mean BMI of $35.5-38.5 \mathrm{~kg} / \mathrm{m}^{2}$. Pioglitazone appears to be effective as well; however, the study is still limited. Although both rosiglitazone and pioglitazone have little short-term risk, fetal safety has not been established (pregnancy category $\mathrm{C}$ of the US FDA guidelines). If used, they should be discontinued as soon as pregnancy has been established. Recently, Tang et al updated the Cochrane review about insulin-sensitizing drugs (metformin, rosiglitazone, pioglitazone, D-chiro-inositol) for women with PCOS, oligo/amenorrhea, and subfertility and concluded that metformin is still of benefit in improving clinical pregnancy and ovulation rates. However, there is no evidence that metformin improves live birth rates whether it is used alone or in combination with clomiphene, or when compared with clomiphene. Therefore, the use of metformin in improving reproductive outcomes in women with PCOS appears to be limited. ${ }^{30}$

\section{Aromatase inhibitors}

Selective aromatase inhibitors such as anastrozole and letrozole are promising new ovulation-inducing agents. They are reversible and highly potent. Unlike $\mathrm{CC}$, which has a half-life of 5-7 days, the mean half-life of anastrozole and letrozole is $\sim 45 \mathrm{~h}$ only. To date, letrozole has been studied much more extensively than anastrozole. ${ }^{31}$ Letrozole was introduced as an assisted reproduction treatment following the appearance of multiple adverse effects of CC, CC's scant therapeutic success, and the complexity of gonadotropin treatment. Letrozole inhibits estrogen production in the hypothalamus-pituitary axis, which implies an increase in gonadotropin-releasing hormone $(\mathrm{GnRH})$ and FSH. It is 
believed that there exists a relative decrease in aromatase in women with PCOS, which reduces the production of follicles responsible for efficacious ovulation. To use this relative deficit, aromatase inhibitors were considered in order to provoke ovulation, because their selective action of blocking the peripheral passage of androgens to estrogens reduces the quantity of estrogens, thereby producing positive feedback in the pituitary, increasing FSH, and optimizing ovulation. The advantage of letrozole is that it avoids peripheral antiestrogenic effects on the endometrium while stimulating monofollicular growth. ${ }^{32}$ Letrozole at $2.5-5 \mathrm{mg}$ is administered for 5 days and may be accompanied by FSH (at the normal doses for PCOS patients) and human chorionic gonadotropin (hCG; 10,000 IU) when the follicle diameter reaches $18 \mathrm{~mm}$ in order to program the ovulation. However, in a prospective randomized trial comparing letrozole with clomiphene, pregnancy rates were similar. Although Novartis Pharmaceuticals (Basel, Switzerland) has warned against the use of letrozole for ovulation induction (owing to possible teratogenicity), a comparison with clomiphene did not demonstrate increased rates of major or minor malformations. ${ }^{33}$

\section{Glucocorticoids}

Glucocorticoids such as prednisone and dexamethasone have been used to induce ovulation. Elnashar et al demonstrated that induction of ovulation by adding dexamethasone (high dose, short course) to CC in CC-resistant PCOS with normal DHEAS is associated with no adverse antiestrogenic effect on the endometrium and higher ovulation and pregnancy rates in a significant number of patients. ${ }^{34}$

In PCOS patients with high adrenal androgen, low-dose dexamethasone $(0.25-0.5 \mathrm{mg})$ at bedtime can be used. ${ }^{35}$ In a study of 230 women with PCOS who failed to ovulate with $200 \mathrm{mg}$ of CC for 5 days, addition of $2 \mathrm{mg}$ of dexamethasone from days 5-14 is associated with a higher ovulation rate and cumulative pregnancy rate. ${ }^{36}$ Enthusiasm for their use is dampened, however, by their potential adverse effects on insulin sensitivity; therefore, prolonged use should be discouraged.

\section{Gonadotropins}

The second possible line of therapy after resistance to $\mathrm{CC}$ has been demonstrated in women with PCOS is exogenous gonadotropins. ${ }^{37}$ The mechanism of action of gonadotropins is to induce ovulation, maintain and provoke optimum follicle growth via the controlled administration of $\mathrm{FSH}$, and achieve a follicle capable of being fertilized. Unlike CC, gonadotropin does not exert a peripheral antiestrogenic effect. The main drawback of gonadotropins is that they provoke multiple follicle development, thereby increasing the risk of ovarian hyperstimulation syndrome (OHSS) and multiple pregnancies. Treatment with FSH is expensive, is time consuming, and requires expertise and stringent monitoring. OHSS is related to hCG-mediated production of vasoactive mediators after gonadotropin-induced multifollicular development. ${ }^{38}$

Several treatment protocols have been advocated, such as step-up, low-dose step-up, and step-down regimens. The ASRM recommends low-dose gonadotropin protocols. ${ }^{39}$ A step-up dose-finding approach favoring unifollicular development is recommended. The step-up regimen starts with a minimum dose (37.5-50 IU/day), which increases according to the lack of follicle response. Control is made by ultrasound, and the regimen is modified after 1 week of no follicle growth with a $50 \%$ increase each time as required. $\mathrm{HCG}$ is used as a surrogate for the LH surge, leading to maturation of the oocyte, rupture of the follicle, and formation of the corpus luteum. The step-down regimen starts with the maximum recommended dose, which is reduced as a follicle response is achieved. The dose is reduced by $50 \%$ each time the regimen is changed. Recent studies have demonstrated greater safety for patients using the step-up regimen. ${ }^{40}$

In 2006, the ASRM advocated caution and strict control when blood estradiol levels exceed $2500 \mathrm{pg} / \mathrm{mL}$ during induction. ${ }^{39}$ Current recommendations suggest withholding hCG administration in the presence of more than two follicles $>16 \mathrm{~mm}$ or more than one follicle $>16 \mathrm{~mm}$ and two additional follicles $>14 \mathrm{~mm}$, or if serum estradiol levels are between 1000 and $2500 \mathrm{pg} / \mathrm{mL}$, particularly in women $<38$ years old without any other infertility factors. Overall, low-dose regimens resulted in a monofollicular ovulation rate of $\sim 70 \%$, a pregnancy rate of $20 \%$ per cycle, and a multiple live birth rate of $5.7 \%$ while maintaining a low incidence of multiple pregnancies $(<6 \%)$ and OHSS $(<1 \%)$. ${ }^{41}$ A maximum of six cycles with gonadotropins is recommended because no response with six cycles signifies resistance.

\section{Laparoscopic ovarian diathermy}

In clomiphene-resistant PCOS women who are unable to comply with the close monitoring necessary for gonadotropin administration, bilateral laparoscopic ovarian surgery with monopolar electrocautery (multiple controlled perforation of the ovary) or laser is an acceptable alternative; both modalities confer similar results. ${ }^{42}$ Laparoscopic ovarian diathermy (LOD) is associated with lower multiple gestation rates than gonadotropins. In a Cochrane Database Systematic Review article, there was no evidence of a 
difference in live birth rate and miscarriage rate in women with clomiphene-resistant PCOS undergoing LOD versus gonadotropin treatment. It appears to be more effective in patients with high $\mathrm{LH}$, and significant reductions in $\mathrm{LH}$ and androgens have been shown following surgery. LOD restores menstrual regularity in $63 \%-85 \%$ of women, and the beneficial effects on reproductive outcomes seem to last for several years in many women. ${ }^{43}$ Treatment with metformin is equally efficacious in correcting the clinical, endocrine, and metabolic abnormalities associated with PCOS. ${ }^{44}$

\section{In vitro fertilization techniques}

The last possibility for achieving a full-term pregnancy in women with PCOS is to use in vitro fertilization (IVF) techniques. ${ }^{6}$ Patients with PCOS are characterized by anovulatory cycles that conceptually are not an indication for IVF techniques. These techniques are used as a last resort when treatments with $\mathrm{CC}$, gonadotropins, and letrozole have failed. IVF is the first choice in cases of concomitant diseases both in women (severe endometriosis, tubal obstruction, etc) and men (azoospermia, male factor) that reduce the effectiveness of other techniques. Because of the increased risk of multiple gestations (up to 10\%) with gonadotropin induction in anovulatory women with PCOS, IVF is a reasonable alternative for women seeking pregnancy. IVF with a single embryo transfer significantly reduced the risk of multiple gestation. ${ }^{45}$ IVF allows the placement of only one embryo or for cryopreservation of all embryos, with transfer of a single embryo in a subsequent cycle with endometrial but not ovarian stimulation IVF.

Several stimulation protocols have been published for the treatment of patients with PCOS undergoing IVF, including combinations or isolated use of clomiphene, human menopausal gonadotropins, recombinant FSH, GnRH agonists, and GnRH antagonists. ${ }^{46,47}$ According to the 2008 ASRM consensus conference, the most commonly employed protocol is a long FSH desensitization protocol in which an agonist is started in the early, mid-, or late luteal phase in the preceding cycle or in the follicular phase until hCG administration. Stimulation with gonadotropins is started when pituitary and ovarian suppression has been achieved. ${ }^{48}$ A meta-analysis published in 2006, which studied the results of conventional IVF techniques in women with PCOS, revealed more cycle cancellation and that the duration of stimulation cycles was significantly longer in women with PCOS. ${ }^{49}$ There is evidence that the use of metformin improves viable pregnancy rates and reduces the incidence of OHSS. ${ }^{50}$ The success of IVF techniques is similar to that of patients without PCOS, which implies that PCOS does not intervene in embryo implantation.

\section{Treatment of menstrual dysfunction}

Chronic anovulation is associated with an increased risk of endometrial hyperplasia and carcinoma. ${ }^{51}$ Thus, it is prudent to consider endometrial biopsy in patients with PCOS who have not had menstrual bleeding for a year or longer. Some investigators have advocated the use of ultrasonography to determine endometrial thickness in deciding whether to do a biopsy of the endometrium. Endometrial proliferation can be inhibited by administering either cyclic progestin or oral contraceptives with a combination of estrogen and progestin. The latter approach, which also reduces ovarian androgen production, may be particularly beneficial in this setting.

\section{Treatment of androgen-related symptoms}

The over-riding androgenic symptoms that the individual presents will vary from patient to patient; for some it is mainly hirsutism, but for others it is acne and/or alopecia. Many have both hirsutism and acne, and a few complain of significant acne, hirsutism, and alopecia. ${ }^{52}$

\section{Hirsutism}

Overall, 70\%-80\% of women with excess androgen demonstrate hirsutism, usually defined as a Ferriman-Gallwey score of at least 8 , although this prevalence is less in certain ethnic groups, such as East Asians, who may have fewer hair follicles endowed per unit area of skin. ${ }^{53}$ Androgens increase the growth rate of hair and transform vellus hair to terminal hair. Reduction of androgens reduces new hair growth and slows the growth of terminal hair that is already present. ${ }^{54}$ Hair grows in nonsynchronous cycles. The growth or anagen phase varies according to body area and is $\sim 4$ months for facial hair. Given this long hair growth cycle, the effects of hormonal therapy require more than 6 months to be maximal.

\section{Oral contraceptive pills}

In women who have no desire to conceive, they can be treated with oral contraceptive pills (OCPs). OCPs reduce hyperandrogenism by promoting direct negative feedback on LH secretion, which results in decreased ovarian synthesis of androgens. Further, they increase liver production of sex hormone-binding globulin and subsequently decrease circulating free androgen. Other mechanisms include reduction in adrenal androgen secretion and inhibition of peripheral conversion of testosterone to dihydrotestosterone and binding of dihydrotestosterone to androgen receptors. ${ }^{55}$ The choice 
of oral contraceptive is important, because most progestins also possess variable androgenic effects. Available low-dose OCPs (defined as $<50 \mu \mathrm{g}$ ) contain ethinyl estradiol in doses ranging from $15 \mu \mathrm{g}$ to $35 \mu \mathrm{g}$. An important consideration for the progestin component is the degree of androgenicity of the progestin. ${ }^{56}$ Newer OCPs contain fewer androgenic progestins (such as norethindrone, desogestrel, and norgestimate), and two progestins (cyproterone acetate [CPA], which is used in low doses in OCPs, and drospirenone) function as androgen receptor antagonists, CPA being more potent in its effect. CPA may also inhibit 5- $\alpha$-reductase activity, decreasing the availability of the more potent androgen, dihydrotestosterone. ${ }^{57} \mathrm{~A}$ third antiandrogenic progestin, dienogest, has recently become available in Europe and is combined with estradiol as an OCP. One of the newest OCPs that might be more effective in reducing the growth of new terminal hair and acne formation is a formula that contains a combination of nonandrogenic progestin, drospirenone, and ethinyl estradiol; thus, it is potentially ideal for the treatment of women with PCOS. Drospirenone is a spironolactone analog with mineralocorticoid activity; as a result, it has some diuretic property. However, it should not be prescribed to those predisposed to hyperkalemia. Treatment must be given for at least 6-9 months before improvement in hirsutism can be seen. ${ }^{56}$

Estrogen-progestin combination therapy (with the use of a combination OCP) remains the predominant treatment for hirsutism and acne in PCOS. ${ }^{4}$ Controversy persists regarding the use of OCPs as first-line therapy in women with PCOS. These agents clearly improve hirsutism and acne and protect against unopposed estrogenic stimulation of the endometrium, but their potential adverse effects on insulin resistance, glucose tolerance, vascular reactivity, and coagulability are a concern, particularly now that insulin-lowering agents are available.

\section{Antiandrogens}

Antiandrogens such as spironolactone, CPA, or flutamide act by competitive inhibition of androgen-binding receptors or by decreasing androgen production. ${ }^{58}$

Spironolactone, which is an aldosterone antagonist, is a dose-dependent competitive inhibitor of the androgen receptor and can also inhibit 5- $\alpha$-reductase activity. Spironolactone possesses moderate antiandrogenic effects when administered in large doses (100-200 mg daily). It has demonstrable effects on hirsutism over and above those induced by OCPs. ${ }^{59}$ Although generally well tolerated, it occasionally causes fatigue, postural hypotension, and dizziness, and when administered alone in high doses, it may cause menstrual irregularity. The risk of feminizing a male fetus, if pregnancy occurs, precludes its use as monotherapy in sexually active women with PCOS. Spironolactone and oral contraceptives appear to be synergistic. ${ }^{60}$ Thus, it is often used with OCPs.

CPA is a progestational antiandrogen. CPA competitively inhibits the binding of testosterone and its more potent conversion product $5 a$-dihydrotestosterone to the androgen receptor. Used in high doses (50-100 mg) and in a reverse sequential regimen (for the first 10 days of cycle), in combination with ethinyl estradiol 20-50 $\mu \mathrm{g}$ (to ensure regular menses), it was shown to be more effective than finasteride, a 5 - $\alpha$-reductase inhibitor. ${ }^{61} \mathrm{CPA}$ is generally well tolerated, although it may cause headaches, nausea, weight gain, breast tenderness, loss of libido, and, rarely, hepatotoxicity effects. As with spironolactone, there is a risk of feminizing a male fetus. A combination of ethinyl estradiol and CPA is very effective in treating hirsutism and acne. Apart from its antiandrogenic effect, CPA has a marked progestational property preventing ovulation. ${ }^{62}$ Loss of hair, which frequently accompanies seborrhea, also improves. Frequent monitoring of liver and renal function is necessary during therapy with antiandrogens. Similar to that with OCP, improvement of hirsutism is expected to be noticeable after 6 months of treatment. Although there is a considerable variation among individuals, the maximum effect is usually seen after 9-12 months of antiandrogen treatment.

Flutamide is a nonsteroidal, selective antiandrogen without progestogenic effect. It is marketed for the treatment of prostate cancer and is very effective in treating hirsutism. In a dose of $500 \mathrm{mg}$ daily, it was found to be similarly effective as spironolactone $100 \mathrm{mg}$ in women with idiopathic hirsutism, and, in a recent study, the minimal effective dose was found to be $125 \mathrm{mg}$ daily. Its major concern is serious hepatotoxicity, although doses up to $375 \mathrm{mg}$ have been used without any significant hepatotoxicity. ${ }^{63}$ However, it is rarely used alone due to its high cost and the risk of hepatocellular toxicity. Recent studies have indicated that a combination of flutamide $62.5 \mathrm{mg}$ daily with metformin $850 \mathrm{mg}$ daily is more effective in improving symptoms of PCOS than OCP alone. ${ }^{64}$

Finasteride is a type 2 (5- $\alpha$-reductase) activity inhibitor that inhibits the production of dihydrotestosterone. Enhanced 5 - $\alpha$-reductase activity in hirsutism probably involves both type 1 and type 2 enzymes, so it is unlikely to be an optimal treatment. Hirsutism scores were lower in studies of finasteride. ${ }^{65}$ Comparison of finasteride with spironolactone has shown equal or lesser efficacy of finasteride. Finasteride has also been used in combination with a CPA-containing OCP, 
and the addition of finasteride $5 \mathrm{mg}$ to the OCP was shown to be better than the OCP alone. ${ }^{66}$ When finasteride was compared directly with an OCP-containing low-dose CPA, the effect was equivalent. Although finasteride has a low side effect profile, its feminizing effects on a male fetus preclude its use in most patients. Due to the risk of feminization of a male fetus, pregnancy must be avoided during treatment with all antiandrogens. ${ }^{67}$

\section{Glucocorticoids}

Some women with PCOS have elevated adrenal androgen levels, although their contribution to ovulatory dysfunction appears modest. ${ }^{68}$ Glucocorticoids suppress adrenal androgen secretion and have been used in patients with adrenal hyperandrogenism. Their use is most legitimate in patients with classic congenital adrenal hyperplasia, where they can help prevent and manage hirsutism and allow ovulatory cycles. In nonclassic congenital adrenal hyperplasia and functional adrenal androgen excess (a minority of PCOS patients), their role is more limited. ${ }^{69}$ Suppression of adrenal androgens results in a minor improvement of hirsutism, although prolonged remission after therapy withdrawal can be obtained. A trial of CPA versus hydrocortisone in patients with late-onset congenital adrenal hyperplasia showed a greater decrease in hirsutism scores with 1 year of CPA compared with hydrocortisone (54\% vs $26 \%$ ). These results occurred despite a greater reduction of androgens with glucocorticoids, highlighting the importance of peripheral receptivity to androgens. ${ }^{70}$ Overdosing can occur, leading to adrenal atrophy, weight gain, and decreased bone mineral density. Glucocorticoid (5-7.5 mg of prednisone once or twice daily) has been shown to improve hirsutism in women with congenital adrenal hyperplasia. However, its effect on hirsutism due to other causes is unclear. ${ }^{65}$ Unless a woman with PCOS has marked adrenal androgen excess, prolonged use of glucocorticoids is not advised.

\section{Gonadotropin-releasing hormone agonist}

Gonadotropin-releasing hormone agonist (GnRHa) is effective even in women with severe insulin resistance who are unresponsive to OCP. ${ }^{71} \mathrm{GnRHa}$ suppresses pituitary hormones, decreases androgen and estradiol secretion, and improves severe forms of hirsutism. To avoid problems associated with estrogen deficiency, 'add-back' therapy with estrogen-progesterone or low-dose OCP is advisable. However, this method of treatment is expensive, limiting its use to severe forms of ovarian hyperandrogenism with hyperinsulinemia.

\section{Insulin-lowering agents}

Both metformin and thiazolidinediones may lower ovarian androgen secretion, mainly through their insulin-lowering effects. In a Cochrane Databse Systematic Review article, limited data on small numbers of patients have shown no evidence of a difference in effect between metformin and OCPs on hirsutism and acne. ${ }^{72}$ Some effects of rosiglitazone on hirsutism were shown by Yilmaz et al and troglitazone (no longer available) improved hirsutism in women with PCOS. ${ }^{72}$

\section{Direct hair removal}

Electrolysis has been used for many years to remove unwanted hair. A fine needle is inserted into the hair follicle, and an electrical current is applied. Erythema and postinflammatory pigment changes may occur, and scarring is possible. ${ }^{73}$ Photoepilation uses laser and nonlaser light sources to damage hair follicles, but vellus hair remains and can be converted to terminal hair. Although laser treatment is more expensive, it is less painful and much faster. ${ }^{74}$ There is potential for depigmentation and scarring with laser use, especially in darker-skinned women.

\section{Topical treatment}

Eflornithine hydrochloride, an inhibitor of the enzyme ornithine decarboxylase in human skin, has been approved for topical use in treating facial hirsutism, taking 6-8 weeks for its effect to be apparent. ${ }^{75}$ It can be combined with laser treatment.

\section{Combination therapy}

In the Endocrine Society Practice Guidelines discussing the evaluation and treatment of hirsutism in premenopausal women, it was recommended that OCPs or direct hair removal be used initially. ${ }^{76}$ Then, if at least 6 months of OCP therapy has not significantly decreased the rate of hair growth, antiandrogens may be added.

\section{Acne}

Both OCPs and antiandrogens have been used successfully in the treatment of acne. ${ }^{77}$ Within 3-6 months of OCP treatment, inflammatory acne counts are reduced by $30 \%-60 \%$, with improvement in 50\%-90\% of patients. OCPs are especially useful in patients with deep-seated nodules and helpful in patients relapsing on isotretinoin.

\section{Alopecia}

There are no extensive trials for alopecia, but OCPs and androgen blockers are usually administered. In limited studies, CPA has had some effect, as has finasteride. ${ }^{78}$ 


\section{Alternative medicine and PCOS}

Absence of evidence is not evidence of absence. Alternative medicine has been emerging as one of the commonly practiced medicines for different health problems. Alternative medicines include many modalities, such as kinesiology, herbalism, homeopathy, reflexology, acupressure, acupuncture, and massage therapy. Acupuncture is the most common modality. The benefit acupuncture seems to have for PCOS sufferers is in helping them regulate and manage their periods. However, it has also been shown to aid in weight loss and reducing headaches as well as improving patients' moods and outlooks. Women with PCOS will have needles placed along the acupuncture meridians related to the reproductive system. This will help stimulate the organs, improve blood flow to the area, contribute to normalizing hormone levels, and promote the proper functioning of the reproductive system.

Because it is only in the last 20 years or so that acupuncture has started to be widely practiced in the West, few studies have been performed on women with PCOS receiving acupuncture. In 2000, a study was carried out by researchers at Göteborg University in Sweden involving 24 women with PCOS who received acupuncture for 2-3 months. At the end of the study, nine women $(38 \%)$ had regular ovulation. However, the study also found that those women with more severe PCOS cases, particularly those participants who had high testosterone and insulin levels and were obese, did not have any luck with the acupuncture treatment. ${ }^{79}$ Recently, a randomized controlled trial proved the efficacy of electroacupuncture in treating women with PCOS. ${ }^{80}$

In conclusion, it is clear that PCOS is an enigma. Its underlying pathophysiology is not fully understood. No treatment is a panacea, because treatments, so far, have been directed at the symptoms but not at the syndrome itself. Extensive efforts should be made to fully investigate the syndrome in order to make therapy more successful and to delay the serious longterm effects of the disease on patients' health.

\section{Disclosure}

The authors report no conflicts of interest in this work.

\section{References}

1. Speroff L, Fritz MA. Anovulation and the polycystic ovary. In: Speroff L, Fritz MA, editors. Clinical Gynecologic Endocrinology and Infertility. 7th ed. Philadelphia (PA): Lippincott Williams \& Wilkins; 2005:470-483.

2. Azziz R. Diagnostic criteria for polycystic ovary syndrome: a reappraisal. Fertil Steril. 2005;83(5):1343-1346.

3. Lakhani K, Prelevic GM, Seifalian AM, Atiomo WU, Hardiman P. Polycystic ovary syndrome, diabetes and cardiovascular disease: risks and risk factors. J Obstet Gynaecol. 2004;24(6):613-621.
4. Ehrmann DA. Polycystic ovary syndrome. NEngl J Med. 2005;352(12): 1223-1236.

5. Dunaif A. Insulin resistance and the polycystic ovary syndrome: mechanism and implications for pathogenesis. Endocr Rev. 1997;18(6): 774-800.

6. Rotterdam ESHRE/ASRM-Sponsored PCOS Consensus Workshop Group. Revised 2003 consensus on diagnostic criteria and long-term health risks related to polycystic ovary syndrome. Fertil Steril. 2004; 81(1):19-25.

7. Azziz R, Carmina E, Dewailly D, et al. Position statement: criteria for defining polycystic ovary syndrome as a predominantly hyperandrogenic syndrome: an Androgen Excess Society guideline. J Clin Endocrinol Metab. 2006;91(11):4237-4245.

8. Adams JM, Taylor AE, Crowley WF Jr, Hall JE. Polycystic ovarian morphology with regular ovulatory cycles: insights into the pathophysiology of polycystic ovarian syndrome. J Clin Endocrinol Metab. 2004; 89(9):4343-4350.

9. Barber TM, Wass JA, McCarthy MI, Franks S. Metabolic characteristics of women with polycystic ovaries and oligo-amenorrhoea but normal androgen levels: implications for the management of polycystic ovary syndrome. Clin Endocrinol (Oxf). 2007;66(4):513-517.

10. Pasquali R, Pelusi C, Genghini S, Cacciari M, Gambineri A. Obesity and reproductive disorders in women. Hum Reprod Update. 2003;9(4): 359-372.

11. Boomsma CM, Eijkemans MJ, Hughes EG, Visser GH, Fauser BC, Macklon NS. A meta-analysis of pregnancy outcomes in women with polycystic ovary syndrome. Hum Reprod Update. 2006;12(6): 673-683.

12. Gjønnaess H. Ovarian electrocautery in the treatment of women with polycystic ovary syndrome (PCOS). Factors affecting the results. Acta Obstet Gynecol Scand. 1994;73(5):407-412.

13. Patel SM, Nestler JE. Fertility in polycystic ovary syndrome. Endocrinol Metab Clin North Am. 2006;35(1):137-155, vii.

14. Reaven GM. The insulin resistance syndrome: definition and dietary approaches to treatment. Annu Rev Nutr. 2005;25:391-406.

15. Bruner B, Chad K, Chizen D. Effects of exercise and nutritional counseling in women with polycystic ovary syndrome. Appl Physiol Nutr Metab. 2006;31(4):384-391.

16. Moran LJ, Brinkworth G, Noakes M, Norman RJ. Effects of lifestyle modification in polycystic ovarian syndrome. Reprod Biomed Online. 2006;12(5):569-578.

17. Escobar-Morreale HF, Botella-Carretero JI, Alvarez-Blasco F, Sancho J, San Millán JL. The polycystic ovary syndrome associated with morbid obesity may resolve after weight loss induced by bariatric surgery. J Clin Endocrinol Metab. 2005;90(12):6364-6369.

18. Homburg R. Clomiphene citrate - end of an era? A mini-review. Hum Reprod. 2005;20(8):2043-2051.

19. Legro RS, Barnhart HX, Schlaff WD, et al. Clomiphene, metformin, or both for infertility in the polycystic ovary syndrome. $N$ Engl J Med. 2007;356(6):551-566

20. Messinis IE. Ovulation induction: a mini review. Hum Reprod. 2005; 20(10):2688-2697.

21. Sam S, Dunaif A. Polycystic ovary syndrome: syndrome XX? Trends Endocrinol Metab. 2003;14(8):365-370.

22. Grundy SM. Obesity, metabolic syndrome, and coronary atherosclerosis Circulation. 2002;105(23):2696-2698.

23. Harborne LR, Sattar N, Norman JE, Fleming R. Metformin and weight loss in obese women with polycystic ovary syndrome: comparison of doses. J Clin Endocrinol Metab. 2005;90(8): 4593-4598.

24. Glueck CJ, Wang P, Goldenberg N, Sieve-Smith L. Pregnancy outcomes among women with polycystic ovary syndrome treated with metformin. Hum Reprod. 2002;17(11):2858-2864.

25. Palomba S, Orio F Jr, Zullo F. What is the best first-step therapeutic approach in treating anovulatory infertility in patients with polycystic ovary syndrome? Questions that are still unanswered. Gynecol Endocrinol. 2007;23(5):245-247. 
26. Moll E, Bossuyt PM, Korevaar JC, Lambalk CB, van der Veen F. Effect of clomifene citrate plus metformin and clomifene citrate plus placebo on induction of ovulation in women with newly diagnosed polycystic ovary syndrome: randomised double blind clinical trial. BMJ. 2006;332(7556):1485.

27. Creanga AA, Bradley HM, McCormick C, Witkop CT. Use of metformin in polycystic ovary syndrome: a meta-analysis. Obstet Gynecol. 2008;111(4):959-968.

28. Hwu YM, Lin SY, Huang WY, Lin MH, Lee RK. Ultra-short metformin pretreatment for clomiphene citrate-resistant polycystic ovary syndrome. Int J Gynaecol Obstet. 2005;90(1):39-43.

29. Azziz R, Ehrmann D, Legro RS, et al. Troglitazone improves ovulation and hirsutism in the polycystic ovary syndrome: a multicenter, double blind, placebo-controlled trial. J Clin Endocrinol Metab. 2001;86(4): $1626-1632$.

30. Tang T, Lord JM, Norman RJ, Yasmin E, Balen AH. Insulin-sensitising drugs (metformin, rosiglitazone, pioglitazone, D-chiro-inositol) for women with polycystic ovary syndrome, oligo amenorrhoea and subfertility. Cochrane Database Syst Rev. 2010;(1):CD003053. Doi: 10.1002/14651858.CD003053.

31. Badawy A, Abdel Aal I, Abulatta M. Clomiphene citrate or letrozole for ovulation induction in women with polycystic ovarian syndrome: a prospective randomized trial. Fertil Steril. 2009;92(3):849-852.

32. Carroll N, Palmer JR. A comparison of intrauterine versus intracervical insemination in fertile single women. Fertil Steril. 2001;75(4): 656-660.

33. Badawy A, Shokeir T, Allam AF, Abdelhady H. Pregnancy outcome after ovulation induction with aromatase inhibitors or clomiphene citrate in unexplained infertility. Acta Obstet Gynecol Scand. 2009;88(2): 187-191.

34. Elnashar A, Abdelmageed E, Fayed M, Sharaf M. Clomiphene citrate and dexamethazone in treatment of clomiphene citrate-resistant polycystic ovary syndrome: a prospective placebo-controlled study. Hum Reprod. 2006;21(7):1805-1808.

35. Nugent D, Vandekerckhove P, Hughes E, Arnot M, Lilford R. Gonadotrophin therapy for ovulation induction in subfertility associated with polycystic ovary syndrome. Cochrane Database Syst Rev. 2000;(4):CD000410. Doi: 10.1002/14651858.CD000410.

36. Parsanezhad ME, Alborzi S, Motazedian S, Omrani G. Use of dexamethasone and clomiphene citrate in the treatment of clomiphene citrate-resistant patients with polycystic ovary syndrome and normal dehydroepiandrosterone sulfate levels: a prospective, double-blind, placebo-controlled trial. Fertil Steril. 2002;78(5):1001-1004.

37. Sastre ME, Prat MO, Checa MA, Carreras RC. Current trends in the treatment of polycystic ovary syndrome with desire for children. Ther Clin Risk Manag. 2009;5(2):353-360.

38. Delbaere A, Smits G, Olatunbosun O, Pierson R, Vassart G, Costagliola S. New insights into the pathophysiology of ovarian hyperstimulation syndrome. What makes the difference between spontaneous and iatrogenic syndrome? Hum Reprod. 2004;19(3):486-489.

39. Christin-Maitre S, Hugues JN; Recombinant FSH Study Group. A comparative randomized multicentric study comparing the step-up versus step-down protocol in polycystic ovary syndrome. Hum Reprod. 2003;18(8):1626-1631.

40. Filicori M, Cognigni GE, Taraborrelli S, et al. Luteinizing hormone activity in menotropins optimizes folliculogenesis and treatment in controlled ovarian stimulation. J Clin Endocrinol Metab. 2001;86(1): 337-343.

41. Maggs DG, Buchanan TA, Burant CF, et al. Metabolic effects of troglitazone monotherapy in type 2 diabetes mellitus. A randomized, double-blind, placebo-controlled trial. Ann Intern Med. 1998;128(3): 176-185.

42. Palomba S, Zullo F, Diamanti-Kandarakis E, Orio F Jr. Surgery and laser diathermy. In: Diamanti-Kandarakis E, Nestler JE, Panidis D, Pasquali R, editors. Insulin Resistance and Polycystic Ovarian Syndrome. Totowa (NJ): Humana Press; 2007: Chap 33, 461-477.
43. Al-Fadhli R, Tulandi T. Laparoscopic treatment of polycystic ovaries: is its place diminishing? Curr Opin Obstet Gynecol. 2004;16(4): 295-298.

44. Palomba S, Orio F Jr, Falbo A, et al. Metformin administration and laparoscopic ovarian drilling improve ovarian response to clomiphene citrate (CC) in oligo-anovulatory CC-resistant women with polycystic ovary syndrome. Clin Endocrinol (Oxf). 2005;63(6):631-635.

45. Van Santbrink EJ, Fauser BC. Is there a future for ovulation induction in the current era of assisted reproduction? Hum Reprod. 2003;18(12): 2499-2502.

46. Dor J, Shulman A, Levran D, Ben-Rafael Z, Rudak E, Mashiach S. The treatment of patients with polycystic ovarian syndrome by in-vitro fertilization and embryo transfer: a comparison of results with those of patients with tubal infertility. Hum Reprod. 1990;5(7):816-818.

47. Griesinger G, Diedrich K, Tarlatzis BC, Kolibianakis EM. GnRH-antagonists in ovarian stimulation for IVF in patients with poor response to gonadotrophins, polycystic ovary syndrome, and risk of ovarian hyperstimulation: a meta-analysis. Reprod Biomed Online. 2006;13(5):628-638.

48. Hamilton-Fairley D, Kiddy D, Watson H, Sagle M, Franks S. Low-dose gonadotrophin therapy for induction of ovulation in 100 women with polycystic ovary syndrome. Hum Reprod. 1991;6(8):1095-1099.

49. Heijnen EM, Eijkemans MJ, Hughes EG, Laven JS, Macklon NS, Fauser BC. A meta-analysis of outcomes of conventional IVF in women with polycystic ovary syndrome. Hum Reprod Update. 2006;12(1): 13-21.

50. Tang T, Glanville J, Orsi N, Barth JH, Balen AH. The use of metformin for women with PCOS undergoing IVF treatment. Hum Reprod. 2006; 21(6):1416-1425

51. Balen A. Polycystic ovary syndrome and cancer. Hum Reprod Update. 2001;7(6):522-525.

52. Nader S. Treatment for polycystic ovary syndrome: a critical appraisal of treatment options. Expert Rev Endocrinol Metab. 2008;3(3): 349-359.

53. Korytkowski MT, Mokan M, Horwitz MJ, Berga SL. Metabolic effects of oral contraceptives in women with polycystic ovary syndrome. J Clin Endocrinol Metab. 1995;80(11):3327-3334.

54. Calaf J, López E, Millet A, et al. Long-term efficacy and tolerability of flutamide combined with oral contraception in moderate to severe hirsutism: a 12-month, double-blind, parallel clinical trial. J Clin Endocrinol Metab. 2007;92(9):3446-3452.

55. Azziz R. Use of combination oral contraceptives in the treatment of hyperandrogenism and hirsutism. UpToDate. Clinical Reference Library. 2006. Available from: http://www.uptodate.com. Accessed Dec 2, 2010.

56. Falsetti L, Gambera A, Tisi G. Efficacy of the combination ethinyl oestradiol and cyproterone acetate on endocrine, clinical and ultrasonographic profile in polycystic ovarian syndrome. Hum Reprod. 2001; 16(1):36-42.

57. Nader S, Diamanti-Kandarakis E. Polycystic ovary syndrome, oral contraceptives and metabolic issues: new perspectives and a unifying hypothesis. Hum Reprod. 2007;22(2):317-322.

58. Falsetti L, Gambera A, Platto C, Legrenzi L. Management of hirsutism. Am J Clin Dermatol. 2000;1(2):89-99.

59. Lobo RA, Shoupe D, Serafini P, Brinton D, Horton R. The effects of two doses of spironolactone on serum androgens and anagen hair in hirsute women. Fertil Steril. 1985;43(2):200-205.

60. Spritzer PM, Lisboa KO, Mattiello S, Lhullier F. Spironolactone as a single agent for long-term therapy of hirsute patients. Clin Endocrinol (Oxf). 2000;52(5):587-594.

61. Venturoli S, Marescalchi O, Colombo FM, et al. A prospective randomized trial comparing low dose flutamide, finasteride, ketoconazole, and cyproterone acetate-estrogen regimens in the treatment of hirsutism. J Clin Endocrinol Metab. 1999;84(4):1304-1310.

62. Rosenfield RL. Clinical practice. Hirsutism. $N$ Engl J Med. 2005; 353(24):2578-2588. 
63. Legro RS, Chiu P, Kunselman AR, Bentley CM, Dodson WC, DunaifA. Polycystic ovaries are common in women with hyperandrogenic chronic anovulation but do not predict metabolic or reproductive phenotype. J Clin Endocrinol Metab. 2005;90(5):2571-2579.

64. Ibáñez L, de Zegher F. Low-dose flutamide-metformin therapy for hyperinsulinemic hyperandrogenism in non-obese adolescents and women. Hum Reprod Update. 2006;12(3):243-252.

65. Lumachi F, Rondinone R. Use of cyproterone acetate, finasteride, and spironolactone to treat idiopathic hirsutism. Fertil Steril. 2003;79(4): 942-946.

66. Bayram F, Müderris II, Sahin Y, Keleștimur F. Finasteride treatment for one year in 35 hirsute patients. Exp Clin Endocrinol Diabetes. 1999; 107(3):195-197.

67. Beigi A, Sobhi A, Zarrinkoub F. Finasteride versus cyproterone acetateestrogen regimens in the treatment of hirsutism. Int J Gynaecol Obstet. 2004;87(1):29-33.

68. Azziz R, Black V, Hines GA, Fox LM, Boots LR. Adrenal androgen excess in the polycystic ovary syndrome: sensitivity and responsivity of the hypothalamic-pituitary-adrenal axis. J Clin Endocrinol Metab. 1998;83(7):2317-2323.

69. Sahin Y, Dilber S, Keleștimur F. Comparison of Diane 35 and Diane 35 plus finasteride in the treatment of hirsutism. Fertil Steril. 2001;75(3):496-500.

70. Spritzer P, Billaud L, Thalabard JC, et al. Cyproterone acetate versus hydrocortisone treatment in late-onset adrenal hyperplasia. J Clin Endocrinol Metab. 1990;70(3):642-646.

71. Cristello F, Cela V, Artini PG, Genazzani AR. Therapeutic strategies for ovulation induction in infertile women with polycystic ovary syndrome. Gynecol Endocrinol. 2005;21(6):340-352.
72. Yilmaz M, Karakoç A, Törüner FB, et al. The effects of rosiglitazone and metformin on menstrual cyclicity and hirsutism in polycystic ovary syndrome. Gynecol Endocrinol. 2005;21(3):154-160.

73. Olsen EA. Methods of hair removal. J Am Acad Dermatol. 1999; 40(2 Pt 1):143-155.

74. Haedersdal M, Wulf HC. Evidence-based review of hair removal using lasers and light sources. J Eur Acad Dermatol Venereol. 2006;20(1): 9-20.

75. Malhotra B, Noveck R, Behr D, Palmisano M. Percutaneous absorption and pharmacokinetics of eflornithine HCL $13.9 \%$ cream in women with unwanted facial hair. J Clin Pharmacol. 2001;41(9): 972-978.

76. Martin KA, Chang RJ, Ehrmann DA, et al. Evaluation and treatment of hirsutism in premenopausal women: an Endocrine Society clinical practice guideline. J Clin Endocrinol Metab. 2008;93(4): 1105-1120.

77. Huber J, Walch K. Treating acne with oral contraceptives: use of lower doses. Contraception. 2006;73(1):23-29.

78. Shapiro J. Clinical practice. Hair loss in women. $N$ Engl J Med. 2007; 357(16):1620-1630.

79. Stener-Victorin E, Waldenström U, Tägnfors U, Lundeberg T, Lindstedt G, Janson PO. Effects of electro-acupuncture on anovulation in women with polycystic ovary syndrome. Acta Obstet Gynecol Scand. 2000;79(3):180-188.

80. Jedel E, Labrie F, Odén A, et al. Impact of electroacupuncture and exercise on hyperandrogenism and oligo/amenorrhoea in women with polycystic ovary syndrome: a randomized controlled trial. Am J Physiol Endocrinol Metab. 2010 Oct 13. Epub ahead of print.
International Journal of Women's Health

\section{Publish your work in this journal}

The International Journal of Women's Health is an international, peerreviewed open-access journal publishing original research, reports, reviews and commentaries on all aspects of women's healthcare including gynecology, obstetrics, and breast cancer. Subject areas include: Chronic conditions (migraine headaches, arthritis, osteoporosis);

\section{Dovepress}

Endocrine and autoimmune syndromes; Sexual and reproductive health; Psychological and psychosocial conditions. The manuscript management system is completely online and includes a very quick and fair peer-review system. Visit http://www.dovepress.com/ testimonials.php to read real quotes from published authors. 